\title{
Die Auswirkungen von Sport auf die Körperzusammensetzung von COPD-Patienten ${ }^{1}$
}

\section{The Effects of Physical Training on the Body Composition of Patients with COPD}

Autoren

Institute
S. A. Jungblut ${ }^{1}$, H. Frickmann ${ }^{2}$, B. Zimmermann ${ }^{3}$, U. Müller ${ }^{3}$, J. Bargon ${ }^{3}$

Medizinische Klinik, Deutsche Klinik für Diagnostik $\mathrm{GmbH}$, Wiesbaden

Institut für Mikrobiologie, Virologie und Hygiene, Universitätsklinikum Rostock

Medizinische Klinik, St. Elisabethen Krankenhaus, Katharina Kasper Kliniken, Frankfurt/Main eingereicht 19.1.2009

akzeptiert nach Revision

7. 4.2009

\section{Bibliografie}

DOI $10.1055 / \mathrm{s}-0029-1214713$

Online-Publikation: 27. 5. 2009

Pneumologie 2009; 63:

374-379 ๔ Georg Thieme

Verlag KG Stuttgart · New York ISSN 0934-8387

Korrespondenzadresse Prof. Dr. Joachim Bargon Medizinische Klinik St. Elisabethen Krankenhaus Ginnheimer Str. 3 60487 Frankfurt/Main bargon@em.uni-frankfurt.de

\section{Zusammenfassung \\ V}

Ziel: Die Studie wurde durchgeführt, um die positiven Effekte von Lungensport auf die Körperzusammensetzung von COPD-Patienten aufzuzeigen.

Methode: Eine Gruppe von 23 COPD-Patienten, die regelmäßig an einem professionell angeleiteten Lungensportprogramm teilnahm, wurde mit einer Kontrollgruppe, bestehend aus 19 nicht sportlich aktiven Patienten, verglichen. Die relativen Veränderungen von Körpermassenindex (BMI), Körperzellmasse in Prozent [BCM-(\%)], Quotient aus Extrazellulärmasse und Körperzellmasse (ECM/BCM-Index) und Phasenwinkel (Winkel zwischen Sinusstrom und Sinusspannung) nach 6 und 12 Monaten wurden zwischen den beiden Gruppen verglichen und statistisch ausgewertet. Ferner und ausschließlich innerhalb der Lungensport treibenden Gruppe wurden die BMI-, BCM-(\%)-, ECM/BCM- und Phasenwinkelergebnisse nach 6, 12 und 18 Monaten mit den zu Beginn der Studie gemessenen Initialwerten verglichen. Die Körperzusammensetzung der Patienten wurde mittels bioelektrischer Impedanzanalyse (Nutriguard-M“-System der Firma „Data Input“) untersucht.

Ergebnisse: Es fanden sich signifikant erhöhte Phasenwinkel- und BCM-(\%)-Werte sowie verringerte $\mathrm{ECM} / \mathrm{BCM}$-Index-Werte bei den Lungensport treibenden COPD-Patienten im Vergleich zu der sportlich nicht aktiven Kontrollgruppe. Ebenfalls konnten signifikante Steigerungen der Parameter BCM-(\%) und Phasenwinkel sowie Verringerungen des ECM/BCM-Indexes innerhalb der Lungensportgruppe gefunden werden, während der BMI-Wert nahezu unverändert blieb. Die akzentuiertesten Verbesserungen waren nach

${ }^{1}$ Teile der Arbeit wurden auf dem 48. Kongress der Deutschen Gesellschaft für Pneumologie und Beatmungsmedizin vorgestellt.

\section{Abstract \\ $\nabla$}

Aim: The following controlled trial was conducted to determine the positive effects of exercise on the body composition of patients suffering from COPD.

Methods: A group consisting of 23 COPD patients who regularly participated in a guided exercise programme was compared with a control group consisting of 19 COPD patients who did not exercise. The relative changes of body mass index (BMI), body cell mass in \% [BCM-(\%)], extra cellular mass/body cell mass index (ECM/ BCM index) and phase angle (angle between sinus current and sinus voltage) after 6 months and after one year were analysed for statistical differences. The values of BMI, BCM-(\%), ECM/BCM index and phase angle at the beginning of the study were compared with the results during the course of the 18 months training merely within the exercising group. The body composition of the patients was determined with the help of the bioelectric impedance analysis (BIA) using the system „Nutriguard M“ produced by „Data Input“.

Results: Significantly raised phase angle values as well as significantly increased BCM-(\%) values and a decreased $\mathrm{ECM} / \mathrm{BCM}$ index were found in the group of patients who exercised compared with the COPD patients who did not exercise. While there were no differences concerning the BMI value, significant increases in BCM-(\%) and phase angle and a significant decrease of the ECM/BCM index could be detected within the group that had been exercising. The best values were recorded after 6 months of exercising. The differences of the group responses resulted from a worsening of the body composition in the control group rather than from improvements in the exercise group.

Conclusion: Physical exercise can improve or at least stabilise the body composition of COPD patients and should be recommended. 
6 Monaten Training zu verzeichnen. Die Unterschiede im Gruppenvergleich basierten mehr auf einer Verschlechterung der Körperzusammensetzung in der Kontrollgruppe als auf einer echten Verbesserung in der Lungensportgruppe.

Schlussfolgerungen: Lungensport kann zu einer Verbesserung oder zumindest Stabilisierung der Körperzusammensetzung von COPD-Patienten beitragen und sollte ihnen daher nahegelegt werden.

\section{Einleitung}

Der Begriff „Lungensport“ beschreibt speziell adaptierte Trainingsprogramme, die darauf abzielen, die körperliche Leistungsfähigkeit von Patienten mit chronischen Lungenerkrankungen wie der COPD zu steigern. Es handelt sich um eine der wenigen Therapieoptionen, die bei COPD-Patienten mit einem signifikanten Anstieg der Lebensqualität einhergehen [1 -3]. Die COPD ist mit pathologischen Stoffwechselveränderungen assoziiert [4], die schließlich dazu beitragen die Prognose zu limitieren. Ein reduzierter Ernährungszustand stellt einen unabhängigen Risikofaktor für Mortalität und Hospitalisation von COPD-Patienten dar [5].

Die Auswirkungen der COPD auf den Ernährungszustand von Körperzellen können mit Hilfe der bioelektrischen Impedanzanalyse (BIA) untersucht werden. Eine Abnahme der aktiven Zellmasse ist indikativ für einen reduzierten Ernährungszustand. Unter solchen Umständen ist die Prognose bei akutem respiratorischen Versagen sehr eingeschränkt [6].

Mit Hilfe der bioelektrischen Impedanzanalyse (BIA) kann der Körper in Kompartimente unterteilt werden: Fettmasse und fettfreie Masse, wobei letztere noch in die Körperzellmasse (BCM) und die Extrazellulärmasse (ECM) untergliedert wird. Der BCMWert subsumiert sowohl Muskel- als auch Parenchymzellen und repräsentiert die Summe aller sauerstoffverbrauchenden, kaliumreichen, Glucose verstoffwechselnden Zellen. Diese Zellen enthalten mehr als 98\% des Gesamtkörperkaliums. Der BCMWert als prozentualer Anteil der fettfreien Körpermasse (BCM(\%)) stellt einen guten Indikator für katabole und anabole Prozesse dar, wenngleich er nicht als direkter Surrogatparameter für die Muskelmasse geeignet ist. Ein guter Ernährungszustand ist durch einen hohen BCM-(\%)-Wert charakterisiert [6]. Die Extrazellulärmasse (ECM) besteht aus festen und flüssigen Anteilen. Das interstitielle Wasser und das Blutplasma bilden den flüssigen Anteil, das Bindegewebe den festen. Im Falle eines guten Ernährungszustands ist die Körperzellmasse immer größer als die Extrazellulärmasse, so dass der ECM/BCM-Index kleiner als 1 sein sollte. Quotienten größer als 1 können Mangelernährung, Ödem oder mangelndes körperliches Training anzeigen. Der Phasenwinkel zwischen maximaler Stromstärke und Spannung eines Sinusstroms liefert weitere Hinweise auf den Zustand der Zellernährung. Ein hoher Phasenwinkel ist typisch für einen guten Ernährungs- und Trainingszustand. Werte unter $4,4^{\circ}-5,7^{\circ}$ gehen zumindest bei Leberzirrhotikern und Kolonkarzinompatienten mit einer ungünstigen Prognose einher [7,8]. Zusammenfassend wird ein guter Ernährungszustand also durch hohe Phasenwinkel- und BCM-(\%)-Werte sowie einen niedrigen ECM/BCM-Index repräsentiert.

Die vorliegende prospektive kontrollierte Studie untersuchte den Einfluss von körperlichem Training auf die Körperzusammensetzung von COPD-Patienten. Ziel war die Erbringung des Nachwei- ses, dass die Körperzusammensetzung durch Lungensport günstig beeinflusst werden kann.

\section{Material, Methoden und Statistik \\ V}

Probanden

Eine Gruppe bestehend aus 23 Patienten mit COPD (13 Männer, 10 Frauen, Altersmedian 68 Jahre, COPD Grad II - III nach GOLD (Global initiative for chronic obstructive lung disease)), die regelmäßig an einem Lungensportprogramm teilnahm, wurde mit einer Kontrollgruppe aus 19 COPD-Patienten verglichen, die sich dem Training nicht unterziehen wollte (13 Männer, 6 Frauen, Altersmedian 71 Jahr, COPD-Grad II - III nach GOLD).

Alle Probanden wurden aus der pneumologischen Abteilung des St. Elisabethen Krankenhauses in Frankfurt am Main rekrutiert. Einziges Verteilungskriterium zur Lungensport- oder Kontrollgruppe war die freiwillige Entscheidung der Patienten, am Training teilzunehmen oder nicht. Unfreiwillige Teilnahme am Lungensport hätte zu keinen repräsentativen Ergebnissen geführt. Ausschlusskriterien waren maligne Tumoren, Immunsuppression sowie COPD-Grade unter II oder über III nach GOLD. Patienten mit COPD des Grades IV wurden von der Studie ausgeschlossen, da eine permanente ärztliche Überwachung der Trainierenden für den Notfall nicht gewährleistet werden konnte. Andere Kriterien zur Beurteilung der Erkrankungsschwere wurden nicht berücksichtigt. Die Lungenfunktions- und Blutgasparameter entsprachen der Definition der COPD Grad II - III nach GOLD, wurden jedoch nicht regelmäßig überprüft. Patienteneigenschaften wie basales Leistungsvermögen, Rauchverhalten, Ernährungsgewohnheiten, durchschnittliche körperliche Aktivität außerhalb des Studienprotokolls, Exazerbations- und Hospitalisationsrate sowie Veränderungen in der medikamentösen Therapie wurden nicht dokumentiert ( Tab. 1).

Tab. 1 Vergleich der Zusammensetzung von Lungensport- und Kontrollgruppe.

\begin{tabular}{|lll|}
\hline & $\begin{array}{l}\text { Lungensportgruppe } \\
\text { Alter (Median) }\end{array}$ & $\begin{array}{l}\text { Kontrollgruppe } \\
78 \text { Jahre }\end{array}$ \\
\hline Geschlechterverhältnis & 13 Männer, 10 Frauen & 13 Männer, 6 Frauen \\
\hline $\begin{array}{l}\text { Schweregrad der } \\
\text { COPD }\end{array}$ & $\begin{array}{l}\text { Grad II-III nach GOLD, jedoch keine } \\
\text { kontinuierliche Lungenfunktionskontrolle im } \\
\text { Studienverlauf }\end{array}$ \\
\hline Rauchverhalten & keine Erfassung im Studienverlauf \\
\hline
\end{tabular}

\section{Studiendesign}

Die Parameter Körpermassenindex (BMI), Körperzellmasse in Prozent (BCM-\%), ECM/BCM-Index und Phasenwinkel der COPDPatienten in Lungensport- und Kontrollgruppe wurden im Rahmen einer kontrollierten Studie erfasst. Ferner wurden diese Parameter innerhalb der Lungensportgruppe im Verlauf der Studie mit den initial gemessenen Werten verglichen.

Das Lungensportprogramm wurde über 18 Monate hinweg begleitet. Über das eigentliche Trainingsprogramm hinaus stand es den Teilnehmern frei, zu Hause nach eigenem Gutdünken weiter zu trainieren.

Die Übungsabläufe beinhalteten sowohl Kraft- als auch Ausdauertraining. Die Patienten trainierten einmal wöchentlich jeweils 90 Minuten unter professioneller Anleitung. Der genaue Aufbau des Lungensportprogramms wurde von Jungblut et al. bereits 
ausführlich beschrieben [9]. Die Intensität der Teilnahme an den einzelnen Übungen konnte von den Teilnehmern nach eigenem Ermessen gewählt werden. Die Compliance der Lungensportgruppe war gut. Im Median wurden 3 von 4 Terminen pro Monat wahrgenommen, wie orientierend aus den Anwesenheitslisten entnommen werden konnte. Ursachen für die Nichtwahrnehmung einzelner Termine, etwa Ausfälle durch Exazerbationen, wurden nicht erfasst.

Alle Studienteilnehmer wurden zu Beginn der Studie sowie nach 6 und 12 Monaten untersucht. Die relativen Veränderungen der Parameter BMI, BCM-(\%), ECM/BCM und Phasenwinkel zwischen Lungensport- und Kontrollgruppe wurden verglichen. Aufgrund der offenkundigen Unterschiede der Mittelwerte der BIA-Parameter der beiden Gruppen wäre ein Vergleich von Absolutwerten nicht sinnvoll gewesen und erfolgte daher nicht. Ebenfalls sprach die hohe Standardabweichung SD innerhalb der einzelnen Gruppen ( $\checkmark$ Tab. 2) gegen ein solches Vorgehen. Aus diesem Grunde wurden die individuellen Veränderungen der Parameter bei den einzelnen Patienten über den Untersuchungszeitraum untersucht. Die Veränderungen wurden für jeden Patienten mit der initialen Messung vor Studienbeginn verglichen. Dieses Vorgehen ermöglichte die Herausarbeitung individueller Verläufe, die bei einer undifferenzierten Betrachtung der Absolutwerte im Hintergrundrauschen der Standardabweichung SD untergegangen wären.

Die Lungensportgruppe wurde nach 18 Monaten Training nochmals untersucht. Eine erneute Untersuchung der Patienten der Kontrollgruppe nach 18 Monaten war aufgrund von Complianceproblemen nicht möglich. Zu viele dieser Probanden verzichteten auf die Teilnahme an der letzten Auswertung. Die Parameter BMI, BCM-(\%), ECM/BCM und Phasenwinkel zum Trainingsbeginn sowie nach 6, 12 und 18 Monaten wurden innerhalb der Lungensportgruppe verglichen. Die Auswirkungen des Trainings auf die körperliche Leistungsfähigkeit wurden nicht quantifiziert.

Die Körperzusammensetzung der Probanden wurde mittels bioelektrischer Impedanzanalyse („Nutriguard M“ System der Firma „Data Input“) erfasst. Die Elektroden des BIA-Messgeräts wurden nach Herstellerangaben an Händen und Füßen angebracht. BCM(\%), Phasenwinkel und ECM/BCM-Index wurden nach den vom Hersteller implementierten Algorithmen berechnet (Data Input, Darmstadt). Da intervenierende Variablen wie Nahrungsaufnahme und körperliche Aktivität die Ergebnisse um mehr als 3\% verfälschen können [10], wurde auf die Standardisierung der Messungen besonderer Wert gelegt. Die Raumtemperatur wurde konstant gehalten. Die Probanden wurden aufgefordert, eine entspannte Position einzunehmen. Die Messungen wurden früh am Morgen zur gleichen Zeit nach einer 10-minütigen Ruhepause durchgeführt, um vergleichbare Ergebnisse zu erhalten. Die Patienten durften während wenigstens 8 Stunden vor der Untersuchung nichts essen und trinken. Auf körperlich anstrengende Tätigkeiten sollte in diesem Zeitraum ebenfalls verzichtet werden. Das Körpergewicht wurde in Unterwäsche mittels einer digitalen Waage mit einer Genauigkeit von $\pm 0,1 \mathrm{~kg}$ bestimmt. Bei der Messung der Körpergröße waren die Probanden barfuß. Die Messgenauigkeit betrug $\pm 0,1 \mathrm{~mm}$.

\section{Statistik}

In der Auswertung wurde nicht zwischen Männern und Frauen unterschieden. Die Veränderungen der untersuchten Parameter in Lungensport- und Kontrollgruppe wurden mittels des T-Tests nach Student mit unterschiedlichen Varianzen verglichen. Die Veränderungen der Parameter BMI, BCM-(\%), ECM/BCM und Pha-

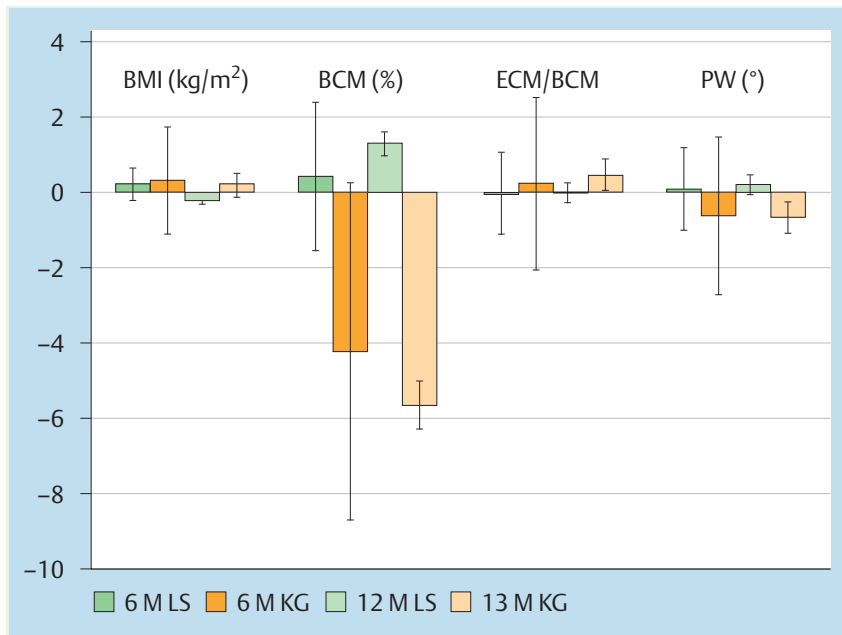

Abb. 1 Individuelle Veränderungen von BMI ( $\left.\mathrm{kg} / \mathrm{m}^{2}\right)$, BCM-(\%), ECM/BCMIndex (ECM/BCM) und Phasenwinkel (PW) $\left(^{\circ}\right)$ im Vergleich der sportlich aktiven Gruppe (LS) mit der Kontrollgruppe (KG) nach 6 Monaten (6 M) und nach 12 Monaten (12 M).

senwinkel innerhalb der Lungensportgruppe wurden mit dem Wilcoxon-matched-pairs-Test untersucht. Die Werte nach 6, 12 und 18 Monaten wurden mit den initialen Messwerten verglichen, um eine Analyse der individuellen Verläufe zu erhalten. Signifikanz wurde ab $\mathrm{p}<0,05$ angenommen, $\mathrm{p}<0,01$ wurde als hoch, $\mathrm{p}<0,001$ als höchst signifikant angesehen.

\section{Ergebnisse \\ $\nabla$}

Zwischen den zwei Gruppen zeigte sich nach 6 Monaten Lungensport kein signifikanter Unterschied in der Entwicklung des Körpermassenindex (BMI) ( $\bullet$ Abb. 1).

Während der Mittelwert in der Lungensportgruppe im Vergleich zu den Initialwerten geringfügig anstieg, war in der Kontrollgruppe ein leichter Abfall zu verzeichnen ( $\bullet$ Tab. 2). Hochsignifikante Unterschiede $(\mathrm{p}<0,01)$ konnten dagegen für die Entwicklungen von Phasenwinkel, Körperzellmasse in Prozent (BCM-[\%]) sowie ECM/BCM-Index demonstriert werden ( $\bullet$ Abb.1). Der Mittelwert des BCM-(\%) war in der Lungensportgruppe im Vergleich zum Initialwert erhöht, in der Kontrollgruppe dagegen erniedrigt. Während sich keine Veränderung des ECM/BCM-Index der Trainingsgruppe im Vergleich zum Studienbeginn fand, war für die Kontrollpopulation ein Anstieg des Mittelwerts des Indexes nachweisbar. Der Phasenwinkel war in der Lungensportgruppe im Mittel in Relation zu den Ausgangwerten erhöht, bei den nicht sportlich aktiven COPD-Patienten dagegen verringert ( Tab. 2).

In beiden Gruppen fanden sich auch nach einem Jahr keine signifikanten Unterschiede hinsichtlich der BMI-Entwicklung, während für $\mathrm{BCM}-(\%)$ und Phasenwinkel hoch signifikante $(\mathrm{p}<0,01)$, für den ECM/BCM-Index signifikante $(p<0,05)$ Entwicklungen in Relation zu den jeweiligen Ausgangswerten aufgezeigt werden konnten $(\bullet$ Abb. 1). Es zeigte sich eine leichte Verringerung des BMI-Mittelwerts in der Übungsgruppe in Relation zur initialen Messung, während in der Kontrollgruppe ein BMI-Anstieg zu verzeichnen war. Der BCM-(\%)-Wert war in der sportlich aktiven Gruppe im Mittel im Vergleich zum Ausgangswert erhöht, während sich in der Kontrollgruppe ein Abfall zeigte. Der Mittelwert des ECM/BCM-Indexes war in der Kontrollgruppe nach 12 Mona- 


\begin{tabular}{|c|c|c|c|c|}
\hline & BMI $(\mathrm{kg} / \mathrm{m} 2) \pm S D$ & $\mathrm{BCM}(\%) \pm \mathrm{SD}$ & $\mathrm{ECM} / \mathrm{BCM} \pm \mathrm{SD}$ & Phasenwinkel $\left({ }^{\circ}\right) \pm \mathrm{SD}$ \\
\hline & \multicolumn{4}{|l|}{ Absolutwerte } \\
\hline $\begin{array}{l}\text { Lungensportgruppe } \\
\text { initial }\end{array}$ & $28,1 \pm 6,6$ & $48,2 \pm 4,2$ & $1,09 \pm 0,19$ & $5,33 \pm 0,72$ \\
\hline $\begin{array}{l}\text { Lungensportgruppe } \\
\text { nach } 6 \text { Monaten }\end{array}$ & $28,1 \pm 6,2$ & $49,8 \pm 4,8$ & $1,03 \pm 0,21$ & $5,64 \pm 0,91$ \\
\hline $\begin{array}{l}\text { Lungensportgruppe } \\
\text { nach } 12 \text { Monaten }\end{array}$ & $27,8 \pm 6,2$ & $49,6 \pm 4,0$ & $1,03 \pm 0,17$ & $5,59 \pm 0,76$ \\
\hline $\begin{array}{l}\text { Lungensportgruppe } \\
\text { nach } 18 \text { Monaten }\end{array}$ & $22,9 \pm 6,4)$ & $49,2 \pm 4,3$ & $1,05 \pm 0,19$ & $5,52 \pm 0,83$ \\
\hline Kontrollgruppe initial & $23,4 \pm 5,7$ & $40,7 \pm 6,7$ & $1,53 \pm 0,45$ & $4,2 \pm 0,95$ \\
\hline $\begin{array}{l}\text { Kontrollgruppe nach } \\
6 \text { Monaten }\end{array}$ & $23,0 \pm 5,8$ & $40,5 \pm 7,2$ & $1,60 \pm 0,50$ & $4,2 \pm 1,03$ \\
\hline \multirow[t]{2}{*}{$\begin{array}{l}\text { Kontrollgruppe nach } \\
12 \text { Monaten }\end{array}$} & $22,4 \pm 5,4$ & $37,4 \pm 10,6$ & $1,97 \pm 0,81$ & $3,6 \pm 1,03$ \\
\hline & \multicolumn{4}{|c|}{ Individuelle Veränderungen im Vergleich zum Studienbeginn } \\
\hline $\begin{array}{l}\text { Lungensportgruppe } \\
\text { nach } 6 \text { Monaten }\end{array}$ & $+0,2 \pm 0,4$ & $+0,4 \pm 1,4$ & $-0,01 \pm 0,09$ & $+0,10 \pm 0,31$ \\
\hline $\begin{array}{l}\text { Lungensportgruppe } \\
\text { nach } 12 \text { Monaten }\end{array}$ & $-0,2 \pm 1,1$ & $+1,3 \pm 2,3$ & $+0,49 \pm 0,27$ & $+0,23 \pm 0,42$ \\
\hline $\begin{array}{l}\text { Kontrollgruppe nach } \\
6 \text { Monaten }\end{array}$ & $+0,3 \pm 2,0$ & $-4,2 \pm 4,5$ & $+0,25 \pm 0,29$ & $-0,61 \pm 0,64$ \\
\hline $\begin{array}{l}\text { Kontrollgruppe nach } \\
12 \text { Monaten }\end{array}$ & $+0,2 \pm 1,1$ & $-5,6 \pm 2,1$ & $0,00 \pm 0,26$ & $-0,65 \pm 0,40$ \\
\hline
\end{tabular}

Tab. 2 Absolutwerte und individuelle Veränderungen der BIA-Parameter.

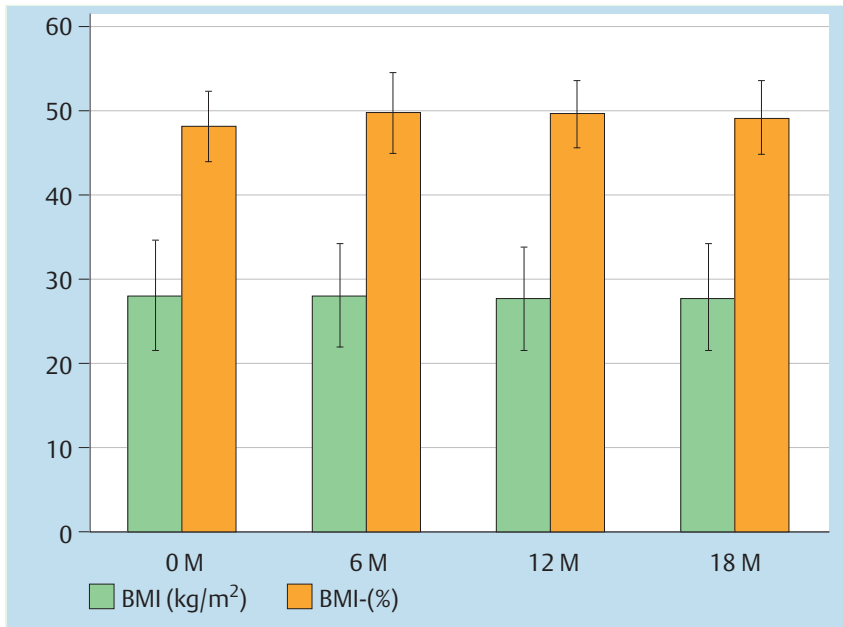

Abb. 2 BMI ( $\left.\mathrm{kg} / \mathrm{m}^{2}\right)$ und BCM-(\%)-Resultate in der Lungensportgruppe nach $0(0 \mathrm{M}), 6(6 \mathrm{M}), 12(12 \mathrm{M})$ und $18(18 \mathrm{M})$ Monaten.

ten im Vergleich zum Initialwert erhöht, während es in der Lungensportgruppe zu keinen nachweisbaren Veränderungen kam. Der Phasenwinkel war bei den regelmäßig trainierenden COPDPatienten im Vergleich zu den Ausgangswerten im Mittel erhöht, in der Kontrollgruppe dagegen reduziert ( $\bullet$ Tab. 2).

Die isolierte Betrachtung der Lungensport treibenden Gruppe über 18 Monate ergab die folgenden Resultate: Der Körpermassenindex (BMI) blieb nach 6 Monaten Lungensport nahezu konstant im Vergleich zu den initialen Messungen. Nach 12 und 18 Monaten Training kam es zu einem Abfall des BMI. Die BMI-Veränderungen waren nach $6(p=0,47), 12(p=0,47)$ und 18 Monaten $(\mathrm{p}=0,57)$ jeweils nicht signifikant $(\bullet$ Abb. 2, $\bullet$ Tab. 2).

Der BCM-(\%)-Wert stieg nach 6 Monaten Lungensport im Vergleich zur initialen Messung hoch signifikant $(p<0.01)$ an. Auch nach einem Jahr Training blieb der Unterschied noch signifikant ( $p<0.05)$, wenngleich eine diskrete Verschlechterung des Mittelwerts zu verzeichnen war. Die Signifikanz ging nach 18 Monaten

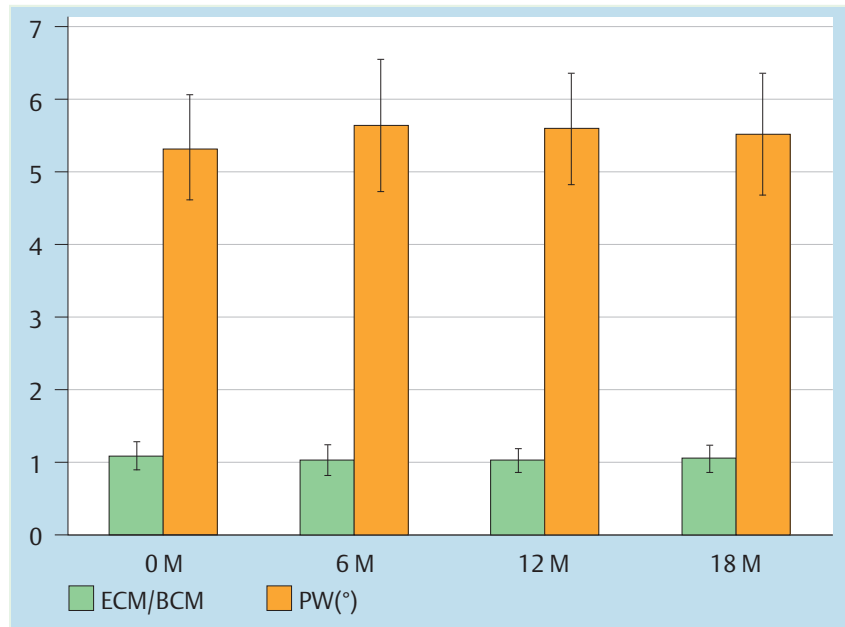

Abb. 3 ECM/BCM-Index (ECM/BCM) und Phasenwinkel (PW) $\left({ }^{\circ}\right)$ in der Lungensportgruppe nach $0(0 \mathrm{M}), 6(6 \mathrm{M}), 12(12 \mathrm{M})$ und 18 (18 M) Monaten.

Lungensport jedoch wieder verloren $(p=0,12)$ ( $\bullet$ Abb. 2, - Tab. 2).

Der ECM/BCM-Index war nach 6 Monaten Lungensport signifikant $(\mathrm{p}<0.05)$ reduziert. Diese Reduktion blieb auch nach einem Jahr noch stabil ( $\mathrm{p}$ 0.05). Nach 18 Monaten kam es allerdings zu einem Anstieg und die Signifikanz im Vergleich zum Ausgangswert ging verloren $(\mathrm{p}=0,22)(\bullet$ Abb. 3, $\bullet$ Tab. 2).

Der Mittelwert des Phasenwinkels war im Vergleich zum Ausgangswert nach 6 Monaten Lungensport hoch signifikant ( $p<0.01$ ) erhöht. Er fiel nach einem Jahr Training wieder, der Anstieg in Relation zum Ausgangswert blieb jedoch hoch signifikant $(\mathrm{p}<0.01)$. Nach 18 Monaten Training war aufgrund einer weiteren Verschlechterung des Mittelwerts keine relative Verbesserung im Vergleich zur initialen Untersuchung $(\mathrm{p}=0,10)$ mehr nachweisbar $($ Abb. 3, $\bullet$ Tab. 2). 


\section{Diskussion}

$\nabla$

Im Rahmen unserer Studie konnten statistisch signifikant positive Entwicklungen von BCM-(\%), Phasenwinkel sowie ECM/BCMIndex bei sportlich aktiven COPD-Patienten im Vergleich zu Patienten, die kein Interesse an Lungensport zeigten, demonstriert werden. Für die BMI-Werte ließ sich kein Unterschied aufzeigen. Die Unterschiede zwischen den Gruppen beruhten mehr auf einer Verschlechterung der untersuchten Parameter in der Kontrollgruppe als auf Verbesserungen in der sportlich aktiven Gruppe. Zumindest konnte eine mittelfristige Stabilisierung der Körperzusammensetzung der COPD-Patienten in der Lungensportgruppe aufgezeigt werden. Ob dies jedoch bereits als therapeutischer Erfolg angesehen werden kann, ist fraglich und müsste durch breiter angelegte Studien und umfassendere Datenerfassung belegt werden. Innerhalb der Lungensportgruppe konnten beste Resultate hinsichtlich BCM-(\%), Phasenwinkel und ECM/ BCM-Index nach 6 Monaten Training erzielt werden, während es danach wieder zu einer Verschlechterung kam. Ob eine individualisierte Intensitätssteigerung beim Training möglicherweise zu deutlicher positiven und anhaltenderen Effekten auf die Körperzusammensetzung geführt hätte, bleibt letztlich unklar. Diese Ergebnisse belegen eine Verbesserung des Trainingszustands von COPD-Patienten durch ein einmal wöchentlich stattfindendes professionell angeleitetes Lungensportprogramm. Durch die im Verlauf der 18 Monate wieder nachweisbare Verschlechterung wird jedoch deutlich, dass der Spontanverlauf der Erkrankung durch das Training nicht völlig aufgehalten werden kann.

Der Mangel an ergänzender Information zur Lebensführung der Patienten stellt einen limitierenden Faktor der Studie dar. Möglicherweise haben die Patienten, die freiwillig am Lungensport teilnahmen, den Spontanverlauf ihrer Erkrankung auch auf andere Weise günstig beeinflusst, beispielsweise durch eine Ernährungsumstellung oder Einschränkungen ihres Nikotinkonsums. Nicht ausgeschlossen werden kann, dass gesündere Lebensweise, höhere Therapieadhärenz und geringerer Tabakkonsum zur günstigeren Entwicklung der Körperzusammensetzung in der Lungensportgruppe beigetragen haben. Auf diese Weise kann die günstige Körperzusammensetzungsentwicklung in der Sportgruppe nicht mit letzter Sicherheit auf die Auswirkungen des Lungensports zurückgeführt werden. Die recht kleine Patientenzahl stellt einen weiteren limitierenden Faktor der Studie dar. Die versäumte ergänzende studienbegleitende Erfassung der Entwicklung der Lungenfunktionsparameter ermöglicht keine Aussagen zur potenziellen diesbezüglichen Beeinflussung des Krankheitsverlaufs durch Lungensport. Der Einfluss von Exazerbationen auf die Körperzusammensetzung kann aufgrund der nicht erfassten Exazerbationsrate ebenfalls nicht abschließend beurteilt werden.

Die Teilnahme am Lungensport kann die Prognose der COPD bekanntermaßen günstig beeinflussen [11,12]. Kontinuierliche mäßige körperliche Belastung trägt zu einer verbesserten Symptomkontrolle bei [13].

Die Teilnahme an Lungensportprogrammen steigert die körperliche Aktivität von COPD-Patienten deutlicher, als dies aufgrund rein physiologischer Modelle zu erwarten wäre. Dies konnte im 6-Minuten-Lauftest und anhand der innerhalb von 6 Minuten in der Tretmühle zurückgelegten Strecke gezeigt werden. Das Phänomen unterstreicht die Bedeutung von verhaltensmodulierend wirksamen Faktoren des Lungensports bei COPD-Patienten [14]. Intensives körperliches Training kann per se eine anabole Reaktion in normalgewichtigen COPD-Patienten induzieren [15]. Mit unserem strukturierten Trainingsprogramm wurde das Voranschreiten kataboler Prozesse zumindest verzögert.

Gewichtsverlust, Mangelernährung und Muskelschwäche sind von großer Bedeutung für die Reduktion der körperlichen Leistungsfähigkeit der COPD-Patienten [16]. Die Körperzusammensetzung beeinflusst unabhängig von der Lungenfunktion das Risiko des Auftretens funktioneller Einschränkungen [17]. Sowohl die Symptome als auch die Prognose werden durch Gewicht und Körperzusammensetzung beeinflusst [5]. Ein niedriger BMI ist mit einer besonders schlechten Prognose assoziiert [18]. Der Körpermassenindex $\left(\mathrm{BMI}=\right.$ Gewicht $[\mathrm{kg}] /$ Höhe $\left.\left[\mathrm{m}^{2}\right]\right)$ allein gestattet jedoch noch keine Rückschlüsse auf die Körperzusammensetzung, die Muskelmasse und den Ernährungszustand [19]. Ein erhöhter ECM/BCM-Index bei gleichzeitig kaum veränderter Fettmasse kann bei COPD-Patienten mit ausgeprägter Reduktion der fettfreien Körpermasse auftreten [20,21]. Dieser Effekt konnte in unserer Kontrollgruppe beobachtet werden.

Die fettfreie Masse limitiert unabhängig von der Atemwegsobstruktion die körperliche Leistungsfähigkeit von Patienten mit schwerer COPD [22]. Ferner erlaubt die fettfreie Masse sehr gute Rückschlüsse auf die periphere Muskelkraft [23], wenngleich sie lediglich einen indirekten Surrogatmarker darstellt. Bekanntermaßen korreliert die körperliche Leistungsfähigkeit von COPDPatienten besser mit der Atemmuskelstärke und Lungenfunktion als mit der Atemnot und Lebensqualität [24]. Eine schwere Depletion der fettfreien Masse führt auch zu einer Abflachung der Atemvolumenkurve bei maximaler körperlicher Leistung, einem reduzierten maximalen Sauerstoffpuls und einem frühen Einsetzen des anaeroben Stoffwechsels bei COPD-Patienten [20,21]. Daher sollte ein Verlust von fettfreier Masse nach Möglichkeit vermieden werden. Strukturierte Übungsprogramme gleich dem beschriebenen können zu diesem Ziel beitragen.

Die COPD führt zu einer generalisierten Muskeldysfunktion [23]. Funktionsstörungen der Extremitätenmuskulatur können unabhängig von Verlusten im Bereich der fettfreien Masse auftreten [25]. Kein Patient unserer Lungensportgruppe berichtete über eine entsprechende Symptomatik.

Während und nach Krankenhausaufenthalten aufgrund von akuten Exazerbationen sind COPD-Patienten betont körperlich inaktiv. Um weitere Verschlechterungen im Bereich der Körperzusammensetzung zu vermeiden, sollte die Patientenführung während und nach akuten Exazerbationsphasen die Ermunterung zu körperlicher Aktivität beinhalten [26]. Die Autoren empfehlen die Initiierung von Lungensportprogrammen, um kritische Krankheitsverläufe zumindest zu verzögern.

Die erhobenen Daten legen positive Auswirkungen von Sport auf die Körperzusammensetzung von COPD-Patienten nahe. Lungensport mildert die schädigenden Auswirkungen der Grunderkrankungen relativ zum Spontanverlauf ab. Für die Parameter BCM (\%), ECM/BCM-Index und Phasenwinkel finden sich sogar diskrete Verbesserungen im Vergleich zum Ausgangswert. Die Studie repräsentiert einen weiteren Hinweis auf die Wirksamkeit von Sport in der COPD-Therapie. 


\section{Literatur}

1 Hui KP, Hewitt AB. A simple pulmonary rehabilitation program improves health outcomes and reduces hospital utilization in patients with COPD. Chest 2003; 124: 94-97

2 Miyahara N, Eda R, Takeyama $\mathrm{H}$ et al. Effects of short-term pulmonary rehabilitation on exercise capacity and quality of life in patients with chronic obstructive pulmonary disease. Acta Med Okayama 2000; 54 : $179-184$

3 Swenson ER. Recommendations for exercise in chronic obstructive pneumopathy (COPD). Ther Umsch 1998; 55: 240-245

4 Müller $U$, Jungblut S, Frickmann $\mathrm{H}$ et al. Assessment of body composition of patients with COPD. Eur J Med Res 2006; 11: 146-151

5 Chailleux E, Laaban JP, Veale D. Prognostic value of nutritional depletion in patients with COPD treated by long-term oxygen therapy: data from the ANTADIR observatory. Chest 2003; 123: 1460 - 1466

6 Faisy C, Rabbat A, Kouchakji B et al. Bioelectrical impedance analysis in estimating nutritional status and outcome of patients with chronic obstructive pulmonary disease and acute respiratory failure. Intensive Care Med 2000; 26: 518-525

7 Gupta D, Lammersfeld CA, Burrows JL et al. Bioelectrical impedance phase angle in clinical practice: implications for prognosis in advanced colorectal cancer. Am J Clin Nutr 2004; 80: 1634 - 1638

8 Selberg $O$, Selberg $D$. Norms and correlates of bio impedance phase angle in healthy human subjects, hospitalised patients, and patients with liver cirrhosis. Eur J Appl Physiol 2002; 86: 509-516

9 Jungblut S, Frickmann H, Klingler J et al. Peak expiratory flow as a predictor for the effectiveness of sport for patients with COPD. Eur J Med Res 2006; 11: 33 - 37

10 Kyle UG, Bosaeus I, De Lorenzo AD et al. Composition of the ESPEN working group. ESPEN GUIDELINES Bioelectrical impedance analysis - part II: utilization in clinical practice. Clinical Nutrition 2004; 23: $1430-1453$

11 Rooyackers J, Jongmans M, Meyer A. Training program for patients with COPD. Pneumologie 1999; 53: 235-236

12 Worth $H$. Why is physical training in patients with COPD valuable? Pneumologie 1999; 53: 231 - 234

13 Rosenberg $H$, Resnick $B$. Exercise intervention in patients with chronic obstructive pulmonary disease (CE). Geriatr Nurs 2003; 24: 90 - 95
14 Behne M, Wewel AR, Kirsten D et al. Exercise training raises daily activity more than predicted from exercise capacity in patients with COPD. Respir Med 2005; 99 (6): 711 - 717

15 Franssen FME, Broekhuizen R, Janssen PP et al. Effects of Whole-Body Exercise Training on Body Composition and Functional Capacity in Normal-Weight Patients with COPD. Chest 2004; 125: 2021 -2028

16 Schols AM. Pulmonary cachexia. Int J Cardiol 2002; 85: 101-110

17 Eisner MD, Blanc PD, Sidney $S$ et al. Body composition and functional limitation in COPD. Respiratory Research 2007; 8: 7

18 Schols AM, Slangen J, Volovics L et al. Weight loss is a reversible factor in the prognosis of chronic obstructive pulmonary disease. Am J Respir Crit Care Med 1998; 157: 1791 - 1797

19 De Benedetto F, Del Ponte A, Marinari S et al. In COPD patients, body weight excess can mask lean tissue depletion: a simple method of estimation. Monaldi Arch Chest Dis 2000; 55: 273-278

20 Baarends EM, Schols AMWJ, Mostert $R$ et al. Peak exercise response in relation to tissue depletion in patients with chronic obstructive pulmonary disease. Eur Respir J 1997; 10: 2807-2813

21 Baarends EM, Schols AM, van Marken Lichtenbelt WD et al. Analysis of body water compartments in relation to tissue depletion in clinically stable patients with chronic obstructive pulmonary disease. Am J Clin Nutr 1997; 65: $88-94$

22 Schols AM, Mostert R, Soeters PB et al. Body composition and exercise performance in patients with chronic obstructive pulmonary disease. Thorax 1991; 46: 695-699

23 Gosker HR, Lencer NHMK, Franssen FME et al. Striking Similarities in Systemic Factors Contributing to Decreased Exercise Capacity in Patients with Severe Chronic Heart Failure or COPD. Chest 2003; 123 $1416-1424$

24 Wijkstra PJ, TenVergert EM, van der Mark TW et al. Relation of lung function, maximal inspiratory pressure, dyspnoea, and quality of life with exercise capacity in patients with chronic obstructive pulmonary disease. Thorax 1994; 49: 468-472

25 Franssen FM, Broekhuizen R, Janssen PP et al. Limb muscle dysfunction in COPD: effects of muscle wasting and exercise training. Med Sci Exercise Exerc 2005; 37: 2 -9

26 Pitta F, Troosters T, Probst VS et al. Physical activity and hospitalisation for exacerbation of COPD. Chest 2006; 129: 536-544 